\title{
DISTRIBUTION AND CONSERVATION IMPLICATIONS OF LIMESTONE PLANT SPECIES IN FELDA CHIKU LIMESTONE FLORA, KELANTAN, MALAYSIA
}

\author{
Kiew R, Ummul-Nazrah AR, Ong PT, Imin K, Aliaa-Athirah AM \& Rafidah AR* \\ Forest Research Institute Malaysia (FRIM), 52109 Kepong, Selangor Darul Ehsan, Malaysia \\ *rafidahar@frim.gov.my \\ Submitted January 2018; accepted June 2018
}

\begin{abstract}
Nationally, the limestone flora is one of the most threatened ecosystems of Peninsular Malaysia. Distribution of the species is a critical factor hampering the conservation of limestone flora. Threatened by the largest cement clinker factory in Southeast Asia, to consume the two largest hills in Federal Land Development Association (FELDA) Chiku oil palm plantation, Kelantan, an intensive survey was initiated to document and provide a permanent record of species richness within the four FELDA Chiku limestone hills and the adjacent one in Relai Forest Reserve. The checklist recorded 266 species of vascular plants in 166 genera and 62 families, of which 27 species are endemic to Peninsular Malaysia, 6 endemic to the state of Kelantan and 3 were site endemics with two new species. The Chiku flora comprised only a fraction (22\%) of the total limestone flora of the Peninsula, i.e. 1,216 species. No single hill had more than $60 \%$ of the 266 species and each of the five hills had a different combination of species. None of the site endemics were found on all five hills, which means no single hill will conserve all the species. Furthermore, 84 species found on the two hills designated for quarrying were not found on the other three hills, contradicting the view that protecting another hill of similar size would protect the entire Chiku limestone flora or that adjacent hills will harbour the same biodiversity. Clearly a network of hills is needed to adequately conserve limestone biodiversity. Hill size, as judged by basal area, did not predict species richness, instead diversity of microhabitats was the most important parameter.
\end{abstract}

Keywords: Biodiversity, microhabitat, oil palm plantation, limestone hills, FELDA

\section{INTRODUCTION}

Limestone hills in Peninsular Malaysia are tower karsts, often with sheer white cliffs rising to about $300 \mathrm{~m}$ that dominate the surrounding landscape. Among the 570 limestone hills in Peninsular Malaysia (Price 2014), the state of Kelantan has the highest number of hills (149 hills, Map 1), but least studied because until the early 1980s they were accessible only by railway line from Kuala Lipis, Pahang to Kuala Krai, Kelantan. Thus, the two plant checklists for the limestone flora of Peninsular Malaysia by Henderson (1939) and Chin (1977, 1979, $1983 a, 1983 b)$ only covered hills adjacent to the railway line. Now that an extensive road network has opened up the hinterland of Kelantan, information on the biodiversity of Kelantan limestone hills is becoming better known (Davison \& Kiew 1990) and several new plant species have come to light, for example, Alocasia farisii (Zulhazman et al. 2017), Bauhinia andersonii (Larsen \& Larsen 1982), Tectaria guachana (Rusea et al. 2004), Begonia tigrina (Kiew 2005), Paraboea nervosissima (Xu \& Burtt 1991), Paraboea lambokensis (Kiew 2010), Impatiens chikuensis, I. glaricola, I. vinosa (Kiew 2016), Microchirita ruthiae (Rafidah \& Haron 2013), Monophyllaea musangensis (Weber 1997) and Vatica najibiana (Ummul-Nazrah et al. 2018).

Kelantan enjoys an equatorial climate. In Gua Musang, mean annual temperatures range between $25.5-28^{\circ} \mathrm{C}$ and annual rainfall averages $2365 \mathrm{~mm}$, distributed throughout the year, with heavier rain towards the end of the year. The climax primary vegetation is rain forest with limestone vegetation, recognised as a distinct edaphic forest type based on a combination of physiognomy and species composition (Saw 2010). It differs from the surrounding lowland rain forest in the near absence of the dominant tree family, Dipterocarpaceae. Crowther (1982), in his study of soil characteristics, noted that the junction between the karst hills and the 
surrounding alluvial plains was often abrupt and that there are significant differences in steepness and soil depth. This is reflected in plant distributions, for example, Tacca integrifolia is a common species in lowland rain forest but in limestone forest it is replaced by Tacca minor. Slopes in the alluvial plain that surrounds the karsts are usually less than $30^{\circ}$ with soils more than $1 \mathrm{~m}$ deep, compared with the steep $40-60^{\circ}$ slopes at the base of karsts where soils are, on average, less than $1 \mathrm{~m}$ deep. Forest on the limestone slopes has a complete canopy but is smaller in stature and the steeper the slope, the less dense and more spindly the trees are. These shallower soils have a lower water holding capacity and are susceptible to periodic water stress, which is a major factor in the exclusion of many species from the surrounding lowland rain forest (Crowther 1982). The soil layer on the craggy summit is found in cracks, crevices and depressions between exposed limestone. Here the trees are of small stature and are twisted and gnarled, and the canopy discontinuous.

The limestone hills occupy $0.4 \%$ of the Peninsula's land area, and the limestone flora is characterised by high species richness. The last plant checklist of limestone flora recorded 1,216 species representing $14 \%$ of the Peninsula's flora of 8000 species, of which $21 \%$ are endemic to Peninsular Malaysia and $11 \%$ are obligate calciphiles restricted to growing on limestone (Chin 1977, 1979, 1983a, 1983b). The species richness is attributed to the diversity of microhabitats stacked on a single hill (Kiew 1991). Different species assemblages grow on large limestone boulders scattered in the surrounding forest, on steep earth slopes up to cliff faces, in gullies and valleys and on rocky scree slopes with accumulated boulders. Vertical cliff faces encompass several microhabitats, i.e. dry deeply shaded overhangs at the base, deeply shaded, humid base of cliffs, wet shaded areas where water regularly drips down, lightly shaded cliff faces and/or dry, hot, fully exposed cliff faces. Caves present several microhabitats, from wet walls to stalactites, and streams with limestone pavement, issuing from the caves. The summits too include a variety of microhabitats, i.e. pockets of soil, cracks and crevices in the craggy limestone where roots can penetrate the exposed limestone rock, and light shade of the tree canopy that supports a rich epiphyte flora.
For most hills, knowledge of the limestone flora is based on single-day botanical surveys. Very few hills, namely, Batu Caves, Selangor and Gunung Kanthan, Perak have been intensively surveyed over a longer period of time, resulting in a published checklist (Kiew 2014, Kiew et al. 2014). From these studies it has become clear that a single hill harbours only about $22 \%$ of the limestone flora. This presents a problem for biodiversity conservation as conserving a single hill will only conserve a fraction of the flora. In addition, many limestone species (192 species) are known from five or fewer hills and these species are distributed among about 80 different hills (Kiew et al. 2017). Individual limestone hills occupy only a small area $\left(<1 \mathrm{~km}^{2}\right.$ in extent) and harbour a high percentage of rare and endangered species, making them vulnerable (Kiew et al. 2017). Threats include quarrying, clearing of the surrounding forest for agricultural activities or urban encroachment that frequently results in uncontrolled burning of limestone vegetation, disturbance of caves from the establishment of temples and resorts, and recreational activities (Kiew 1997).

Saw et al. (2009) considered limestone vegetation as one of the most threatened forest types. Majority of limestone hills do not have protected status. Very few are protected within Taman Negara (Kelantan, Pahang and Terengganu), State Parks (Perlis), Geoparks (Langkawi, Kedah) or Protection Forest Reserves, and even fewer are Archaeological Museum Sites (e.g. Lenggong, Perak). Of the 149 hills in Kelantan, only some small outcrops that lie within Taman Negara are protected. The vulnerability of limestone biodiversity is recognised nationally. Under the National Physical Plan (2010), limestone hills are categorised as environmentally sensitive areas ranked 1. For major threats or activities, such as quarrying, an environmental impact assessment is mandatory. Limestone hills were also proposed as important plant areas (Saw et al. 2009) and the Ministry of Natural Resources \& Environment Malaysia 2016 highlighted limestone as one of Malaysia's most vulnerable ecosystems (National Policy on Biological Diversity 2016-2025).

Internationally, the limestone ecosystem has long been recognised as threatened and in 
need of protection. The International Union for Conservation of Nature (IUCN) produced 'Guidelines for Cave \& Karst Protection' that outline conservation needs for karst sites, emphasising the importance of selecting limestone hills with high biodiversity and minimal degradation, that need to be clearly delimited and be given secure legal protection with a conservation management plan that includes regular review (Watson et al. 1997).

The IUCN Guidelines also emphasise the importance of awareness of conservation and other values like tourism and recreation (Kiew 1997). In Peninsular Malaysia, karst hills generally lie on state land. Until recently, the extractive value of limestone took precedence over cave formations and fossils, but now landscape and other values are being appreciated. Several areas, namely Langkawi, Kedah and Kinta Valley, Perak, have been declared Geoparks, though Geopark status is not as secure as protection within State or National Parks.

Corlett (2016) highlighted the urgent need globally to complete botanical inventory and assessments of conservation status so that conservation can be targeted effectively. Darbyshire et al. (2017) highlighted the frequent lack or incompleteness of information in tropical regions, not only on species, but also on habitats. Vermeulen \& Whitten (1999), with specific reference to limestone resources, emphasise the need for systematic inventories to provide an atlas of plant species distributions and to identify endangered species. A major impediment in Peninsular Malaysia is the lack of sound scientific data on the distribution of plant species on limestone (Kiew et al. 2017). This has increasingly become a critical issue, to balance exploitation of limestone for cement and other commercial products required for development with conservation of limestone ecosystem. Recommendations for conserving the limestone ecosystem have been advocated, such as the requirement of a forested buffer zone around the base of the hill and advocating the exploitation of below-ground limestone (Davison \& Kiew 1990, MNS 1990). Clements et al. (2008) suggested that larger hills are more species rich and should be targets for conservation. Vermeulen \& Whitten (1999) advocated giving priority to isolated or remote hills, based on the assumption that an endemic species is unlikely to be restricted to just one part of a large conservation area. Thus, it is better to site a quarry in areas with long continuous limestone hills rather than creating many small quarries on several different hills.

In 2014, two limestone hills in Federal Land Development Association (FELDA) Chiku were approved for quarrying as a source of limestone for a cement clinker factory. Forest Research Institute Malaysia (FRIM) undertook to improve the knowledge of their flora by:

a) carrying out an intensive survey of these poorly known hills to make a permanent record of their flora before FELDA Chiku 7 (Ktn 66) and 8 (Ktn 67) were totally destroyed by quarrying, and

b) providing detailed checklists for the individual hills.

In addition, being the first detailed study of a group of limestone hills, it provided evidence for queries with implications on conservation management of limestone flora:

1. Do adjacent or nearby hills share the same level of species richness and site endemics, rare and endangered species?

2. Will conserving FELDA Chiku 4 (Ktn 49) compensate for the loss of biodiversity when FELDA Chiku 7 (Ktn 66) and 8 (Ktn 67) are quarried, as was asserted by ChemsainKonsultant Sdn. Bhd. (2015)?

3. Are hill size and/or distance between them relatively important determinants of both species richness and endemism as suggested for land snails (Clements et al. 2008)?

4. Are site endemics widespread on the hill where they are found, as suggested by Vermeulen \& Whitten (1999)?

5. How important is the buffer zone around the base of the hill for conservation of plant species, as was suggested by Davison \& Kiew (1990)?

\section{Chiku limestone}

The Chiku limestone lies about $48 \mathrm{~km}$ north of Gua Musang, Gua Musang District, Kelantan, and is accessed by the Gua Musang to Kuala Krai highway. The area only became accessible in the late 1980s when lowland forest was converted to palm oil plantation by FELDA. FELDA Chiku takes its name from the Sungai Chiku (Chiku River) and the limestone hills are commonly known by the areas designated by FELDA (Table 1 ), however to avoid confusion it is advocated using the numbers assigned by Price (2014). 
Table 1 The Chiku limestone hills, Kelantan, their location and size

\begin{tabular}{|c|c|c|c|c|}
\hline Hill number & Local name & GPS & Dates surveyed & Size $\left(\mathrm{km}^{2}\right)$ \\
\hline Ktn 49 & FELDA Chiku 4 & $5^{\circ} 03.69^{\prime} \mathrm{N}, 102^{\circ} 11.62^{\prime} \mathrm{E}$ & $10 \& 11$ Oct 2017 & 1.3247 \\
\hline Ktn 50 & Relai FR & $5^{\circ} 02.81^{\prime} \mathrm{N}, 102^{\circ} 11.83^{\prime} \mathrm{E}$ & $19 \& 20$ Oct 2015 & 0.2933 \\
\hline Ktn 64 & $\begin{array}{l}\text { Gua Harimau } \\
\text { Gua Tok Guru }\end{array}$ & $5^{\circ} 01.61^{\prime} \mathrm{N}, 102^{\circ} 09.49^{\prime} \mathrm{E}$ & $\begin{array}{l}23 \text { April, } \\
13 \text { \& } 15 \text { May, } \\
18 \text { \& } 21 \text { Oct } 2015\end{array}$ & 0.3581 \\
\hline Ktn 66 & FELDA Chiku 7 & $5^{\circ} 02.55^{\prime} \mathrm{N}, 102^{\circ} 09.23^{\prime} \mathrm{E}$ & $\begin{array}{l}\text { 21, } 22 \& 24 \text { April, } \\
13 \text { \& } 14 \text { May } 2015\end{array}$ & 0.7605 \\
\hline Ktn 67 & FELDA Chiku $7 \& 8$ & $\begin{array}{l}5^{\circ} 04.58^{\prime} \mathrm{N}, 102^{\circ} 09.42^{\prime} \mathrm{E} \\
5^{\circ} 03.81^{\prime} \mathrm{N}, 102^{\circ} 08.95^{\prime} \mathrm{E} \\
5^{\circ} 04.03^{\prime} \mathrm{N}, 102^{\circ} 08.74^{\prime} \mathrm{E} \\
5^{\circ} 03.93^{\prime} \mathrm{N}, 102^{\circ} 09.05^{\prime} \mathrm{E}\end{array}$ & $\begin{array}{l}12 \text { May, } 14 \text { May, } \\
31 \text { Aug, } 1 \text { Sept } 2015\end{array}$ & 1.4237 \\
\hline
\end{tabular}

GPS = global positioning system

Following modern spelling, Chiku is sometimes written as Ciku, however Chiku is used on the topographical map taken from Series L 7030.

Prior to our study, the only biodiversity survey carried out there was that by Davison \& Kiew (1990), who visited FELDA Chiku 4, 5 and 8 . They reported that lowland forest had been cleared right to the base of the hills and that there was no buffer zone of trees, and only remnants of the limestone forest at the foot of the cliffs remained. It was clear, from the thick covering of creepers on the cliffs, that large areas of hill vegetation had been accidentally burned when fire was used to clear the felled forest for plantation. In many places, only vegetation on the vertical flanks and summits was still pristine. On the smaller hills (Ktn 68, 70, 71 and 72) creepers smothered the entire hill, even the summit. This study included a preliminary list of plants. Specimens from the survey were deposited in the Kepong (KEP) and Singapore (SING) herbaria.

Clements et al. (2008) collected snails from FELDA Chiku 7 and subsequently Tan (2015) reported that Clements considered one snail from FELDA Chiku 7 to be an undescribed new species, while Mohammad Effendi Marzuki was reported to have found two new snails species from FELDA Chiku 4.

Sinar Harian Online (2014) reported the ground-breaking ceremony officiated by the Chief Minister of Kelantan for the establishment of the largest cement clinker production line in Malaysia, with a daily capacity of 10,000 tonnes of clinker by the Australian ASN Cement Company, that would quarry two hills, FELDA Chiku 7 (Ktn 66) and FELDA Chiku 8 (Ktn 67) (Tan 2015). The Kelantan State government gave the assurance that the establishment of the cement factory would not affect the 'national treasures' found on Chiku limestone hills (Utusan Online 2015).

Subsequently the Preliminary (EIA) and Detailed Environmental Impact Assessments (DEIA) were submitted to the Department of the Environment (Chemsain Konsultant, 2015). Caves, cave fauna, archaeology and limestone flora were not satisfactorily covered by DEIA, but a new species of bent-toed gecko was discovered in a cave in FELDA Chiku 7. Chemsain-Konsultant (2015) reported they were unable to access the summit but relied on identification with binoculars. Their plant lists lacked information on the general limestone flora, and rare and endemic species were not investigated. Apparently specimens were not collected, so no permanent or verifiable record of their survey exists. They concluded that the flora of Chiku 7 (Ktn 66) and Chiku 4 (Ktn 49) were similar and recommended conserving Chiku 4. However, it was not specified whether it should be a condition of the license or which agency would undertake the legal protection.

Until today, only a preliminary study of caves has been carried out by the local caving community and none has been mapped nor have the neolithic artefacts in Ktn 71, estimated to be 10,000 years old, been investigated (Tan 2015). The hills have not been afforded any protection, and currently, hunting for serow (Capricornis sumatranus), a protected species, appears to be rife as judged from the number of traps encountered and information from local guides. Free-ranging cattle and commercial orchid collectors impact on the flora at the base of the hills. 


\section{MATERIALS \& METHODS}

\section{Location}

The Chiku limestone lies about $48 \mathrm{~km}$ north of Gua Musang, Gua Musang District, Kelantan. There are approximately eight limestone hills (Ktn 49, Ktn 64, Ktn 65, Ktn 66, Ktn 67, Ktn 68, Ktn 70 and mykarst-080) within the FELDA Chiku area (Map1).

The numbering/naming of the limestone hills is based on Price (2014) and Liew et al. (2016) through the Google Earth interface. Other limestone hills lie mostly within the Relai Forest Reserve (FR) (i.e. Ktn 47, Ktn 48, Ktn 50, Ktn 71 and Ktn 72). The number of limestone hills may or may not be precise because some hills break up into smaller outcrops, some of which are very small (Price 2014). Ktn 67, on the other hand, is a big hill which lies between the boundary of Chiku 7 and Chiku 8, hence it is not separated. The hills are of various sizes (Table 1). Most limestone hills within FELDA Chiku are adjacent to each other (200-800 m apart), while some are isolated (approximately $4 \mathrm{~km}$ apart). $\mathrm{Ktn} 50$ on the topographical map is shown as surrounded by Relai Forest Reserve (FR), but in fact is now surrounded by palm oil plantation.
The FRIM botany team spent 18 days conducting intensive field surveys of the flora of limestone hills in FELDA Chiku and the Relai FR between 2015 and 2017 (Table 1).

\section{Field survey}

Traditional quantitative methods, such as using quadrats or transects, have proved to be ineffective or misleading on limestone because the terrain is so varied with many microhabitats within a small area. It is difficult, if not impossible, to find areas where repeatable quadrats or transects can be set up, thus, many microhabitats fall outside the quadrats or transects and many rare and endangered nichespecific species are not captured. For example, a few species grow only on stalactites that hang from the cave mouth.

The field surveys were therefore carried out in such a way as to cover all types of terrain with special attention paid to accessing all microhabitats. Climbing limestone is dangerous, thus, a few microhabitats, such as vertical cliff faces, were inaccessible. Experienced guides were necessary to reach the summit safely, sometimes requiring the use of ropes. Intensity of collection was limited by time, but after two

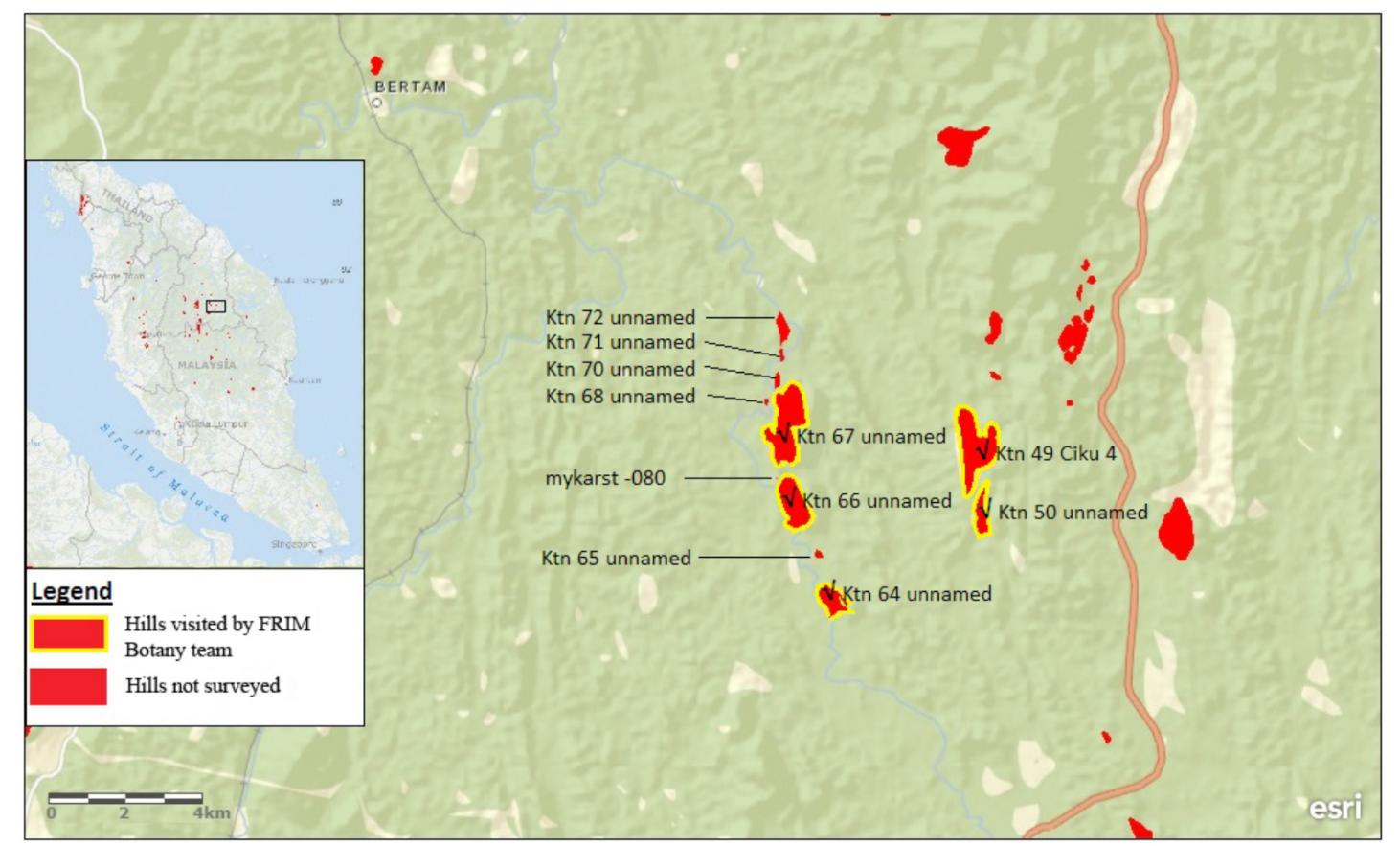

Map 1 Limestone hills within FELDA Chiku and Relai Forest Reserve, Gua Musang District, Kelantan; Hill number and/or name in yellow were hills visited by the FRIM botany team between 2015-2017 
or three days' collecting the number of species, not previously collected, declined rapidly.

\section{Specimen collection}

This checklist is specimen-based, all species were collected at first sighting. Fertile specimens deposited in the KEP herbarium (Thiers 2017) were individually numbered using the Forest Research Institute (FRI) number series, with data recorded in the KEP field collection book. Sterile plant material was also collected and made into voucher specimens. The plants were pressed, dried and mounted on paper to serve as a permanent record (Bridson \& Forman 1992; Chung et al. 2009). In addition, spirit collections of flowers and fruits were made. Specimen data were accessioned in the Botanical Research and Herbarium Management System (BRAHMS). For vouchers (sterile specimens) in an electronic file is available from FRIM. The photographic record included images of the habit, flowers and fruits. Weed species were excluded.

Living collections were also made and planted in the KEP nursery in FRIM. Epiphytes (e.g. orchids and ferns) were mounted on wood slabs cushioned with a thin layer of sphagnum moss, while terrestrials or lithophytes (e.g. begonias, balsams, aroids and orchids) were potted up using either soil mix (50\% soil: $50 \%$ perlite) or rocks cushioned with a thin layer of sphagnum moss, the medium varying depending on plant type. Epiphytic plants were generally placed under $50 \%$ (or less) shade net whereas understory plants were placed under $80 \%$ shade net. Plants with specialised microclimate needs (e.g. high humidity) were placed in either a transparent ziplock bag or a terrarium (transparent container). Once established, they were then removed from the ziplock bag or container.

\section{Species identification}

Identification of specimens was made by consulting the literature and by comparing the specimens with those in the KEP herbarium. The main references used were Chin $(1977,1979$, 1983a, 1983b), the Flora of the Malay Peninsula (Ridley 1922-1923, 1924a, 1924b, 1925), the Tree Flora of Malaya (Whitmore 1972, 1973; Ng
1978, 1989), Flora of Peninsular Malaysia (Kiew et al. 2010; 2011, 2012, 2013, 2015, 2017; Parris et al. 2010, 2013), Wayside Trees of Malaya (Corner 1988) and The Orchids of Peninsular Malaysia and Singapore (Seidenfaden \& Wood 1992). Turner (1997) was used for information on species distribution. Not all specimens, for example, if they lacked flowers or fruits, could be identified, and so these were treated as taxonomic units and labelled as sp. 1, sp. 2, etc. to give an indication of their diversity.

\section{Assessment of conservation status}

The regional conservation status (extinction risk) assessment is based on the IUCN Red List Categories and Criteria version 3.1 (IUCN 2012). Nine categories are recognised: Extinct (EX), Extinct in the Wild (EW), Critically Endangered (CR), Endangered (EN), Vulnerable (VU), Near Threatened (NT), Least Concern (LC), Data Deficient (DD) and Not Evaluated (NE). The assessment was based on the information contained in the Taxon Data Information Sheet (TDIS) stored in FRIM. The TDIS comprises scientific name, taxonomy details, common names, habitat preferences, geographical range, general distribution pattern, population decline, threats, Red List Categories and Criteria, a rationale for the listing, current conservation measures, utilisation, literature used in assessment, details of assessor(s), date of assessment and names of evaluators. Regional conservation assessment is currently ongoing for plant species of Peninsular Malaysia with about $20 \%$ of species already assessed. (MyBIS, website www.mybis.gov.my).

Endemic species are defined as being restricted to a particular place, e.g. to Peninsular Malaysia or to Kelantan. Site endemics are those species known from a single limestone hill.

\section{RESULTS}

\section{Species richness}

During the botanical survey, 266 species of vascular plants in 166 genera and 62 families were collected, of which 228 are flowering plants, 2 are gymnosperms, 28 ferns and 8 lycophytes (Table 2). All the species listed in Davison \& Kiew (1990) were recollected. 
Table 2 Checklist of vascular plant species collected from limestone hills in FELDA Chiku and Relai Forest Reserve, Kelantan

\begin{tabular}{|c|c|c|c|c|c|}
\hline & Ktn 49 & Ktn 50 & Ktn 64 & Ktn 66 & Ktn 67 \\
\hline \multicolumn{6}{|l|}{ Lycophytes } \\
\hline \multicolumn{6}{|l|}{ Lycopodiaceae } \\
\hline Phlegmariurus carinatus & + & & & & + \\
\hline Phlegmariurus phlegmaria & & & & & + \\
\hline Phlegmariurus squarrosus & + & & & & + \\
\hline Phlegmariurus tetrastichus & + & & & & \\
\hline \multicolumn{6}{|l|}{ Selaginellaceae } \\
\hline Selaginella alutacia & + & & & & \\
\hline Selaginella mayeri & + & + & + & & + \\
\hline Selaginella minutifolia & & & + & & \\
\hline Selaginella padangensis & + & + & + & & + \\
\hline \multicolumn{6}{|l|}{ Gymnosperms } \\
\hline \multicolumn{6}{|l|}{ Cycadaceae } \\
\hline Cycas clivicola & & & & + & + \\
\hline \multicolumn{6}{|l|}{ Gnetaceae } \\
\hline Gnetum sp. & & + & & & \\
\hline \multicolumn{6}{|l|}{ Ferns } \\
\hline \multicolumn{6}{|l|}{ Adiantaceae } \\
\hline Antrophyum callifolium & & + & + & & + \\
\hline Antrophyum parvulum & + & & & + & \\
\hline Haplopteris elongata & + & & & & \\
\hline \multicolumn{6}{|l|}{ Aspleniaceae } \\
\hline Asplenium batuense & & & + & & \\
\hline Asplenium macrophyllum & + & + & & & + \\
\hline Asplenium nidus & & + & & & \\
\hline Asplenium pellucidum & + & & & & \\
\hline Asplenium salignum & + & + & + & + & \\
\hline Asplenium unilaterale & & + & + & & \\
\hline \multicolumn{6}{|l|}{ Dryopteridaceae } \\
\hline Bolbitis heteroclita & & + & & & \\
\hline \multicolumn{6}{|l|}{ Lomariopsidaceae } \\
\hline Cyclopeltis crenata & & + & & & \\
\hline \multicolumn{6}{|l|}{ Loxogrammaceae } \\
\hline Loxogramme avenia & & & & & + \\
\hline Loxogramme scolopendrioides & + & + & & & + \\
\hline Loxogramme subecostata & & & & & + \\
\hline \multicolumn{6}{|l|}{ Polypodiaceae } \\
\hline Drynaria sparsisora & & & & & + \\
\hline Microsorum membranifolium & & & + & & + \\
\hline Microsorum scolopendria & & + & & & + \\
\hline Pyrossia lanceolata & & & & & + \\
\hline Pyrrosia nummulariifolia & + & + & & & + \\
\hline Pyrrosia penangiana & + & & & & + \\
\hline \multicolumn{6}{|l|}{ Pteridaceae } \\
\hline Pteris longipinnula & & + & + & & + \\
\hline Pteris vittata & & + & & & \\
\hline \multicolumn{6}{|l|}{ Tectariaceae } \\
\hline Heterogonium nayarii & & + & + & & + \\
\hline Tectaria griffithii & & & + & & \\
\hline Tectaria keckii & + & + & + & & + \\
\hline Tectaria stenosemioides & + & & & & \\
\hline \multicolumn{6}{|l|}{ Thelypteridaceae } \\
\hline Pneumatopteris truncata & & & & & + \\
\hline Pronephrium triphyllum & & & & & + \\
\hline Flowering plants & & & & & \\
\hline
\end{tabular}


Table 2 Continued

\begin{tabular}{|c|c|c|c|c|c|}
\hline & Ktn 49 & Ktn 50 & Ktn 64 & Ktn 66 & Ktn 67 \\
\hline \multicolumn{6}{|l|}{ Acanthaceae } \\
\hline Gymnostachyum sp. nov ${ }^{\mathrm{EK}}$ & + & + & & & \\
\hline Gymnostachyum sp. 1 & + & & & & \\
\hline Gymnostachyum sp. 2 & + & & & & \\
\hline Justicia grossa & & & & & + \\
\hline Justicia ptychostoma ${ }^{\mathrm{E}}$ & & + & + & + & \\
\hline \multicolumn{6}{|l|}{ Annonaceae } \\
\hline Anaxagorea javanica & + & & + & & \\
\hline Artabotrys sp. & & & & & + \\
\hline Cananga odorata & & & & & + \\
\hline Goniothalamus subevenius & & & & & + \\
\hline Goniothalamus sp. 1 & & & + & & \\
\hline Goniothalamus sp. 2 & & & & & + \\
\hline Goniothalamus sp. 3 & & & + & & \\
\hline Meiogyne kanthanensis ${ }^{\mathrm{E}}$ & & & & + & + \\
\hline Miliusa intermedia & & & & + & \\
\hline Orophea chrysantha ${ }^{\mathrm{EK}}$ & & & & + & \\
\hline Orophea cuneiformis & + & & + & + & + \\
\hline Orophea cumingiana ${ }^{\mathrm{E}}$ & & + & & & \\
\hline Orophea enterocarpa & & & + & & + \\
\hline Orophea hirsuta & + & + & + & + & + \\
\hline Orophea sp. 1 & & & & & + \\
\hline Orophea sp. 2 & & + & & & \\
\hline Polyalthia sp. 1 & + & & & + & \\
\hline Polyalthia sp. 2 & & + & & & + \\
\hline Pseuduvaria setosa & + & & & & \\
\hline Uvaria sp. & & & & & + \\
\hline \multicolumn{6}{|l|}{ Actinidiaceae } \\
\hline Saurauia pentapetala & & & & & + \\
\hline \multicolumn{6}{|l|}{ Araceae } \\
\hline Aglaonema simplex & & & & + & + \\
\hline Alocasia farisi $^{\mathrm{EK}}$ & + & + & + & + & + \\
\hline Alocasia longiloba & + & & & & \\
\hline Amorphophallus carneus ${ }^{\mathrm{E}}$ & & & & & + \\
\hline Amorphophallus elegans ${ }^{\mathrm{E}}$ & & & & & + \\
\hline Amorphophallus prainii & & & & + & + \\
\hline Arisaema roxburghii & + & & & + & + \\
\hline Arisaema sp. nov. ${ }^{\mathrm{E}}$ & + & & & & + \\
\hline Cryptocoryne sp. & + & & & & \\
\hline Hapaline brownii & + & + & & + & \\
\hline Homalomena humilis & + & + & + & & + \\
\hline Lasia spinosa & & & + & & \\
\hline Pothos macrocephalus & & & & & + \\
\hline Rhaphidophora sp. & & & & + & \\
\hline Schismatoglottis wallichii & & & & & + \\
\hline Schismatoglottis calyptrata & & & & & + \\
\hline Typhonium sp. & + & & & & \\
\hline \multicolumn{6}{|l|}{ Araliaceae } \\
\hline Schefflera sp. & & & & & + \\
\hline \multicolumn{6}{|l|}{ Aristolochiaceae } \\
\hline Thottea piperiformis & + & & & & \\
\hline \multicolumn{6}{|l|}{ Apocynaceae } \\
\hline Dischidia sp. 1 & & & & & + \\
\hline Dischidia sp. 2 & + & & & & \\
\hline Heterostemma piperifolium & + & & & & \\
\hline Hoya coronaria & & & & & + \\
\hline Hoya sp. 1 & & & & & + \\
\hline
\end{tabular}


Table 2 Continued

\begin{tabular}{|c|c|c|c|c|c|}
\hline & Ktn 49 & Ktn 50 & Ktn 64 & Ktn 66 & Ktn 67 \\
\hline Hoya sp. 2 & & & & & + \\
\hline \multicolumn{6}{|l|}{ Balsaminaceae } \\
\hline Impatiens foxworthyi $\mathrm{E}^{\mathrm{E}}$ & + & + & & + & + \\
\hline Impatiens chikuensis ${ }^{\mathrm{EK}}$ & + & + & & & + \\
\hline \multicolumn{6}{|l|}{ Begoniaceae } \\
\hline Begonia foxworthyi ${ }^{\mathrm{E}}$ & & & + & + & + \\
\hline Begonia kingiana ${ }^{\mathrm{E}}$ & + & + & + & & \\
\hline Begonia nurii ${ }^{\mathrm{E}}$ & + & + & & & + \\
\hline \multicolumn{6}{|l|}{ Celastraceae } \\
\hline Microtropis bivalvis & & & & & + \\
\hline \multicolumn{6}{|l|}{ Chloranthaceae } \\
\hline Chloranthus erectus & & + & + & & + \\
\hline \multicolumn{6}{|l|}{ Commelinaceae } \\
\hline Amischotolype monosperma ${ }^{\mathrm{E}}$ & & & & + & \\
\hline Pollia thyrsiflora & & & + & & \\
\hline \multicolumn{6}{|l|}{ Convallariaceae } \\
\hline Peliosanthes teta & & & + & & \\
\hline \multicolumn{6}{|l|}{ Dioscoreaceae } \\
\hline Dioscorea scortechinii & & & + & & \\
\hline Dioscorea sp. 1 & + & & & & \\
\hline Dioscorea sp. 2 & & + & & & \\
\hline \multicolumn{6}{|l|}{ Dipterocarpaceae } \\
\hline Vatica najibiana ${ }^{\mathrm{E}}$ & & + & & & \\
\hline \multicolumn{6}{|l|}{ Dracaenaceae } \\
\hline Dracaena cf. chiniana & + & & & & \\
\hline Dracaena porter $^{\mathrm{E}}$ & & & + & & \\
\hline Dracaena sp. & + & & & & \\
\hline \multicolumn{6}{|l|}{ Ebenaceae } \\
\hline Diospyros cauliflora & & + & & & \\
\hline Diospyros sp. 1 & + & & & & \\
\hline Diospyros sp. 2 & & + & & & \\
\hline Diospyros sp. 3 & & & & + & \\
\hline \multicolumn{6}{|l|}{ Elaeocarpaceae } \\
\hline Elaeocarpus floribundus & & + & & & \\
\hline \multicolumn{6}{|l|}{ Euphorbiaceae } \\
\hline Cladogynos orientalis & & & & & + \\
\hline Croton argyratus & & & & & + \\
\hline Euphorbia sp. 1 & & + & + & & \\
\hline Euphorbia sp. 2 & & & + & & \\
\hline Mallotus cf. peltatus & & & + & & \\
\hline Mallotus montanus & & & & & + \\
\hline Mallotus subpeltatus & & & + & & \\
\hline Suregada multiflora & & + & & & \\
\hline \multicolumn{6}{|l|}{ Gesneriaceae } \\
\hline Damrongia lacunosa & + & & & & + \\
\hline Epithema membranaceum ${ }^{\mathrm{E}}$ & + & & + & & + \\
\hline Epithema saxatile & + & & + & & \\
\hline Epithema sp. nov. ${ }^{\mathrm{E}}$ & & + & + & & \\
\hline Microchirita caliginos $a^{\mathrm{E}}$ & + & + & & & \\
\hline Microchirita involucrata & + & + & & & \\
\hline Monophyllaea hirticalyx ${ }^{\mathrm{E}}$ & + & & + & & \\
\hline Monophyllaea horsfieldii & + & + & + & + & + \\
\hline Paraboea sp. nov. ${ }^{\mathrm{EK}}$ & + & & & & \\
\hline Paraboea nervosissima ${ }^{\mathrm{E}}$ & & & & & + \\
\hline Stauranthera grandiflora & & & + & & + \\
\hline \multicolumn{6}{|l|}{ Hypoxidaceae } \\
\hline Molineria sp. & + & & & & \\
\hline
\end{tabular}


Table 2 Continued

\begin{tabular}{|c|c|c|c|c|c|}
\hline & Ktn 49 & Ktn 50 & Ktn 64 & Ktn 66 & Ktn 67 \\
\hline \multicolumn{6}{|l|}{ Labiatae } \\
\hline Paraphlomis javanica & & & + & & + \\
\hline \multicolumn{6}{|l|}{ Lauraceae } \\
\hline Endiandra macrophylla & & & + & & \\
\hline Litsea cordata & & & + & & \\
\hline \multicolumn{6}{|l|}{ Leeaceae } \\
\hline Leea indica & & + & & & \\
\hline \multicolumn{6}{|l|}{ Leguminosae } \\
\hline Bauhinia intergifolia & & & + & & \\
\hline Bauhinia scortechinii ${ }^{\mathrm{E}}$ & & & + & & \\
\hline Bauhinia sp. & & & & & + \\
\hline Cassia sp. & & + & & & \\
\hline \multicolumn{6}{|l|}{ Loranthaceae } \\
\hline Macrosolen formosus & & + & & & \\
\hline \multicolumn{6}{|l|}{ Maesaceae } \\
\hline Maesa sp. & & & & & + \\
\hline \multicolumn{6}{|l|}{ Malvaceae } \\
\hline Grewia laevigata & & + & & & \\
\hline Hibiscus macrophyllus & + & & & & \\
\hline \multicolumn{6}{|l|}{ Marantaceae } \\
\hline Donax grandis & & & + & & \\
\hline \multicolumn{6}{|l|}{ Meliaceae } \\
\hline Aglaia sp. 1 & & & & & + \\
\hline Aglaia sp. 2 & & & & & + \\
\hline \multicolumn{6}{|l|}{ Memecylaceae } \\
\hline Memecylon cantleyi & & & + & & \\
\hline Memecylon sp. 1 & + & & & & \\
\hline Memecylon sp. 2 & & & + & & \\
\hline Memecylon sp. 3 & & & & + & \\
\hline \multicolumn{6}{|l|}{ Moraceae } \\
\hline Ficus sp. 1 & & & & & + \\
\hline Ficus sp. 2 & & & & & + \\
\hline Ficus sp. 3 & & & & & + \\
\hline Ficus sp. 4 & + & & & & \\
\hline Streblus ilicifolia & + & & & & \\
\hline \multicolumn{6}{|l|}{ Myrsinaceae } \\
\hline Ardisia subverticillata & & & + & & \\
\hline Ardisia sp. 1 & + & & & & \\
\hline Ardisia sp. 2 & + & & & & \\
\hline Ardisia sp. 3 & + & & & & \\
\hline Ardisia sp. 4 & + & & & & \\
\hline \multicolumn{6}{|l|}{ Oleaceae } \\
\hline Jasminum insigne & & + & & & \\
\hline \multicolumn{6}{|l|}{ Orchidaceae } \\
\hline Adenoncos major & + & & & & \\
\hline Appendicula torta & + & & & & + \\
\hline Arachnis cf. flos-aeris & & & & & + \\
\hline Brachypeza pallida & & & & & + \\
\hline Bulbophyllum flammuliferum & & + & & & + \\
\hline Bulbophyllum mirum & & & & & + \\
\hline Calanthe ceciliae & + & & & & \\
\hline Calanthe triplicata & & + & & & + \\
\hline Campanulorhis leiophylla & & & & & + \\
\hline Cleisostoma discolor & + & & & & + \\
\hline Cleisostoma javanicum & & + & & & \\
\hline Cleisostoma nieuwenhuisii & + & + & & & \\
\hline
\end{tabular}


Table 2 Continued

\begin{tabular}{|c|c|c|c|c|c|}
\hline & Ktn 49 & Ktn 50 & Ktn 64 & Ktn 66 & Ktn 67 \\
\hline Cleisostoma scortechinii & + & + & & & + \\
\hline Coelogyne asperata & + & + & & & + \\
\hline Coelogyne cf. foerstermannii & & & & & + \\
\hline Corybas cf. calcicola ${ }^{\mathrm{E}}$ & & + & & & \\
\hline Corymborkis veratrifolia & + & & & & \\
\hline Crepidium calophyllum & + & + & & & + \\
\hline Crepidium rheedei & & & & & + \\
\hline Dendrobium crumenatum & + & & & & \\
\hline Dendrobium hendersonii & + & + & & & + \\
\hline Dendrobium panduriferum & & & & & + \\
\hline Dendrobium plicatile & + & & & & + \\
\hline Dendrobium rhodostele & + & + & & & + \\
\hline Dendrobium setifolium & + & & & & \\
\hline Dienia ophrydis & & + & & & \\
\hline Eria javanica & & & & & + \\
\hline Eria jenseniana & + & + & & & \\
\hline Eria mucronata & + & + & & & + \\
\hline Eulophia graminea & & + & & & \\
\hline Goodyera hispida & + & & & & + \\
\hline Grosourdya appendiculata & + & & & & + \\
\hline Habenaria reflexa & & & & & + \\
\hline Hetaeria oblongifolia & & + & & & + \\
\hline Liparis cespitosa & + & & & & + \\
\hline Liparis viridicallus & & + & & & + \\
\hline Liparis viridiflora & & & & & + \\
\hline Microsaccus ampullaceus & & & & & + \\
\hline Nervilia punctata & & & & & + \\
\hline Oberonia cf. transversiloba & & & & & + \\
\hline Oxystophyllum carnosum & + & & & & \\
\hline Phalaenopsis deliciosa & & & & & + \\
\hline Phalaenopsis cf. sumatrana & + & & & & + \\
\hline Pholidota imbricata & + & + & & & + \\
\hline Podochilus microphyllus & + & & & & \\
\hline Pomatocalpa kunstleri & + & & & & + \\
\hline Pomatocalpa spicata & & & & & + \\
\hline Renanthera elongata & + & + & & & \\
\hline Robiquetia minimiflora & & & & & + \\
\hline Schoenorchis secundiflora & & & & & + \\
\hline Stichorkis cf. calcicola & & & & & + \\
\hline Taeniophyllum stella & & & + & & \\
\hline Thelasis micrantha & + & & & & + \\
\hline Thelasis pygmaea & + & + & & & + \\
\hline Trachoma rhopalorrhachis & & & & & + \\
\hline Trichoglottis celebica & & & & & + \\
\hline Trichoglottis retusa & & + & & & + \\
\hline Trichoglottis ventricularis & & & & & + \\
\hline \multicolumn{6}{|l|}{ Palmae } \\
\hline Arenga westerhoutii & + & + & + & + & + \\
\hline Calamus diepenhorstii & & & & + & \\
\hline Licuala sp. & + & + & & & \\
\hline \multicolumn{6}{|l|}{ Pandanaceae } \\
\hline Pandanus irregularis ${ }^{\mathrm{E}}$ & + & + & + & + & + \\
\hline \multicolumn{6}{|l|}{ Phyllanthaceae } \\
\hline Antidesma velutinosum & & & & & + \\
\hline Baccaurea racemosa & & & & & + \\
\hline Baccaurea sp. & & & + & & \\
\hline
\end{tabular}


Table 2 Continued

\begin{tabular}{|c|c|c|c|c|c|}
\hline & Ktn 49 & Ktn 50 & Ktn 64 & Ktn 66 & Ktn 67 \\
\hline Bridelia sp. 1 & & & & & + \\
\hline Bridelia sp. 2 & & & + & & \\
\hline Leptopus australis & & + & & & + \\
\hline Phyllanthus reticulatus & & & & & + \\
\hline Phyllanthus sp. & & & & & + \\
\hline \multicolumn{6}{|l|}{ Piperaceae } \\
\hline Peperomia dindygulensis & & & & + & \\
\hline Piper kurzii & & & & + & \\
\hline \multicolumn{6}{|l|}{ Polygalaceae } \\
\hline Polygala cf. malesiana & & + & & & \\
\hline \multicolumn{6}{|l|}{ Rubiaceae } \\
\hline Canthium sp. & & & + & & \\
\hline Chassalia chartacea & & & & + & \\
\hline Ixora pendula & & & + & + & \\
\hline Ixora sp. & & & & & + \\
\hline Lasianthus sp. & & & + & & \\
\hline Morinda umbellata & & & & & + \\
\hline Mycetia cauliflora & & & + & & \\
\hline Ophiorrhiza remotiflora & + & + & & & + \\
\hline Ophiorrhiza discolor & & + & + & & \\
\hline Pavetta sp. & & & + & & \\
\hline Psychotria rostrata & & & + & & \\
\hline Psychotria sp. & & & + & & \\
\hline Saprosma sp. & & + & & & \\
\hline Tarenna sp. & & & + & & \\
\hline Urophyllum sp. & & & & + & \\
\hline \multicolumn{6}{|l|}{ Rutaceae } \\
\hline Melicope sp. & & & + & & \\
\hline \multicolumn{6}{|l|}{ Salicaceae } \\
\hline Flacourtia sp. & & & & & + \\
\hline \multicolumn{6}{|l|}{ Stemonuraceae } \\
\hline Gomphandra quadrifida & & & + & & \\
\hline \multicolumn{6}{|l|}{ Sterculiaceae } \\
\hline Firmiana malayana & & & + & & \\
\hline \multicolumn{6}{|l|}{ Taccaceae } \\
\hline Tacca integrifolia & & & & & + \\
\hline Tacca minor & + & + & + & & + \\
\hline \multicolumn{6}{|l|}{ Urticaceae } \\
\hline Elatostema cyrtandrifolium & & & & & + \\
\hline Elatostema latifolium & + & & + & & \\
\hline Elatostema sessile & + & + & + & + & + \\
\hline Pilea fruticosa & + & + & & & + \\
\hline Procris pedunculata & + & & & & + \\
\hline \multicolumn{6}{|l|}{ Violaceae } \\
\hline Rinorea bengalensis & + & + & & + & \\
\hline Rinorea horneri & + & + & + & & + \\
\hline \multicolumn{6}{|l|}{ Zingiberaceae } \\
\hline Boesenbergia cf. longipes ${ }^{\mathrm{E}}$ & & & & + & \\
\hline Globba sp. & + & & & & \\
\hline Zingiber sp. & & + & & & \\
\hline
\end{tabular}

${ }^{\mathrm{E}}=$ species is endemic in Peninsular Malaysia, ${ }^{\mathrm{EK}}=$ endemic in Kelantan 


\section{Species of conservation importance}

\section{Endemic species}

Twenty seven species (about $10.2 \%$ of the 266 species) are endemic in Peninsular Malaysia (Table 2). Among these, six are Kelantan endemics: three (Orophea chrysantha, Annonaceae; Alocasia farisii, Araceae; Epithema sp., Gesneriaceae) occur on other limestone hills elsewhere in Kelantan, while three (Gymnostachyum sp., Acanthaceae; Paraboea sp., Gesneriaceae and Impatiens chikuensis, Balsaminaceae) are site endemics, to date known only in Chiku limestone. The new species recently discovered by FRIM survey have yet to be named and described scientifically. The number of new species discovered is an indication that the flora of Kelantan is still relatively poorly known.

\section{Threatened and/or endangered species}

Site endemics that grow on Ktn 66 and Ktn 67 are threatened by extinction from quarrying, while those on other Chiku hills are under threat of habitat disturbance, since none of the hills has legal protection. For those species for which an assessment is available, three are critically endangered, nine are endangered and nine are vulnerable. All species listed in Table 3 are restricted to limestone habitats.

\section{Differences between the Chiku hills}

There are great differences in species richness between hills, that relate neither to hill size nor to collecting intensity. Ktn 49 and Ktn 67 are almost the same size $\left(1.3-1.4 \mathrm{~km}^{2}\right)$ but Ktn 67 has almost one and a half times more species (Table 4). Similarly Ktn 66 is twice the size of Ktn 50 and Ktn 64 but has less than half the number of species. With reference to collecting effort, Ktn 50 (70 species) and Ktn 64 (81 species) are of similar size but twice the collecting effort on Ktn 64 netted fewer species.

\section{DISCUSSION}

In common with the other detailed surveys of limestone hills in Peninsular Malaysia, it is clear that an individual limestone hill harbours only a fraction, about 15-25\%, of Peninsular Malaysia's limestone flora (Kiew 2014; Kiew et al. 2014). The total figure for all Chiku limestone hills is 266 species, which represents $22 \%$ of Peninsular Malaysia limestone flora. The most species rich hill, Ktn 67 with 135 species, harboured 50.7\% of the Chiku flora representing $11.4 \%$ of the Peninsula's of the 266 species (Table 4 ).

The intensive survey illustrated that for hills, even $5 \mathrm{~km}$ apart, and presumably originally part of a larger hill or part of the same complex of hills, there are significant differences between them, not only in number of species but also in species composition (Table 2). This carries implications for conservation management because it cannot be assumed that by conserving one hill in an area, all the species of that area will be conserved. For example, it was suggested by Chemsain Konsultant (2015) that conserving Chiku 4 (Ktn 49) would conserve the same biodiversity that is found on Chiku 7 (Ktn 66) and 8 (Ktn 67), which will be destroyed by quarrying. However, the current study showed that 84 species recorded from these two hills were not found on Ktn 49 (Table 2). This demonstrated that intensive, competent biodiversity surveys need to be carried out before decisions are made as to which hills can be exploited.

Where intensive surveys have been carried out (Batu Caves, Gunung Kanthan and the Chiku limestone), representation of speciesrich families is similar to that of the general limestone flora for Peninsular Malaysia (Table 5). The most species-rich families in Chiku limestone (Table 5) are Orchidaceae (37 genera, 58 species), followed by Annonaceae (10 genera, 20 species), Araceae (12 genera, 17 species), Rubiaceae (12 genera, 15 species) and Gesneriaceae (6 genera, 11 species). Orchids on all hills are particularly species-rich, which is to be expected since Orchidaceae is the largest family in Peninsular Malaysia with 972 species (Ong et al. 2017). The relatively higher figure for Chiku limestone is in part attributed to collecting sterile plants, which are nurtured to flowering in the nursery, thereby allowing species identification.

Differences at species level, in part, reflect phytogeographic differences (Kiew 1991, Kiew et al. 2017). The Chiku limestone hills fall within the region termed the Continental Intrusion, an area that covers the limestone hills in Kelantan and Pahang as compared with the 
Table 3 Threatened species on FELDA Chiku limestone

\begin{tabular}{|c|c|c|c|c|c|}
\hline Species & Family & $\begin{array}{c}\text { Conservation } \\
\text { status }\end{array}$ & $\begin{array}{c}\text { Endemic to } \\
\text { Peninsular Malaysia }\end{array}$ & $\begin{array}{l}\text { Endemic to } \\
\text { Kelantan }\end{array}$ & $\begin{array}{l}\text { Endemic to Chiku } \\
\text { Limestone }\end{array}$ \\
\hline Alocasia farisii & Araceae & EN & + & + & \\
\hline Amorphophallus carneus & Araceae & VU & + & & \\
\hline Begonia kingiana & Begoniaceae & $\mathrm{EN}$ & + & & \\
\hline Begonia nurii & Begoniaceae & VU & + & & \\
\hline Cleisostoma discolor & Orchidaceae & $\mathrm{VU}$ & & & \\
\hline Cleisostoma nieuwenhuisii & Orchidaceae & CR & & & \\
\hline Corybas cf. calcicola & Orchidaceae & EN & + & & \\
\hline Cycas clivicola & Cycadaceae & $\mathrm{EN}$ & + & & \\
\hline Damrongia lacunosa & Gesneriaceae & EN & & & \\
\hline Epithema membranaceum & Gesneriaceae & VU & + & & \\
\hline Epithema sp. nov. & Gesneriaceae & VU & + & + & \\
\hline Gymnostachyuum sp. nov. & Acanthaceae & EN & + & + & + \\
\hline Impatiens chikuensis & Balsaminaceae & CR & + & + & + \\
\hline Impatiens foxworthyi & Balsaminaceae & VU & + & & \\
\hline Meiogyne kanthanensis & Annonaceae & CR & + & & \\
\hline Pandanus irregularis & Pandanaceae & VU & + & & \\
\hline Paraboea nervosissma & Gesneriaceae & VU & + & & \\
\hline Paraboea sp. nov. & Gesneriaceae & $\mathrm{EN}$ & + & + & + \\
\hline Phalaenopsis deliciosa & Orchidaceae & VU & & & \\
\hline Phalaenopsis sumatrana & Orchidaceae & $\mathrm{EN}$ & & & \\
\hline Vatica najibiana & Dipterocarpaceae & $\mathrm{EN}$ & + & & \\
\hline
\end{tabular}

IUCN categories: $\mathrm{CR}=$ critically endangered, $\mathrm{EN}=$ endangered, $\mathrm{VU}=$ vulnerable to extinction

Table 4 Hill size, number of species, percentage representation of the total Chiku flora and collecting effort

\begin{tabular}{lcccc}
\hline Hill & $\begin{array}{c}\text { Area } \\
\left(\mathrm{km}^{2}\right)\end{array}$ & $\begin{array}{c}\text { Number of } \\
\text { species }\end{array}$ & $\begin{array}{c}\text { Number of } \\
\text { survey days }\end{array}$ & $\begin{array}{c}\text { Percentage representation of } \\
\text { total flora }\end{array}$ \\
\hline Ktn 49 & 1.30 & 99 & 2.0 & 37.2 \\
Ktn 50 & 0.30 & 81 & 2.0 & 30.5 \\
Ktn 64 & 0.35 & 70 & 4.5 & 26.3 \\
Ktn 66 & 0.76 & 33 & 4.5 & 12.4 \\
Ktn 67 & 1.40 & 135 & 4.0 & 50.7 \\
\hline
\end{tabular}

Table 5 The most species-rich families on karst limestone in Peninsular Malaysia

\begin{tabular}{lrlrlrlr}
\hline Peninsular Malaysia & No. spp. & Chiku limestone & No. spp. & Batu Caves & No. spp. Gunung Kanthan & No. spp. \\
\hline Orchidaceae & 136 & Orchidaceae & 58 & Orchidaceae & 23 & Araceae & 17 \\
Euphorbiaceae s.l. & 81 & Annonaceae & 20 & Apocynaceae s.l. & 20 & Orchidaceae & 14 \\
Rubiaceae & 66 & Araceae & 17 & Rubiaceae & 14 & Annonaceae & 11 \\
Araceae & 44 & Gesneriaceae & 11 & Araceae & 12 & Rubiaceae & 10 \\
Apocynaceae s.l. & 40 & Rubiaceae & 15 & Gesneriaceae & 9 & Gesneriaceae & 9 \\
Annonaceae & 39 & Euphorbiaceae s.l. & 8 & Moraceae & 9 & Euphorbiaceae & 8 \\
Gesneriaceae & 39 & Acanthaceae & 5 & Urticaceae & 9 & Acanthaceae & 4 \\
Total Number of & 1216 & & 266 & & 269 & & 195 \\
Species & & & & & & & \\
\hline
\end{tabular}


Northern Region (Perlis and Kedah) and the Perak Region (Perak and Selangor). Limestone species characteristic of the Continental Intrusion include Begonia nurii, Impatiens foxworthyi, Paraboea nervosissima and Pandanus irregularis.

\section{Endangered species}

All three site endemic species are endangered because they are known from very few localities within the FELDA Chiku area, have an extremely small area of occupancy and are threatened by habitat destruction from quarrying. An additional 19 species are assessed as threatened (Table 3). However, this is a poor reflection of conservation status because, apart from one or two genera, none of the species-rich families like the Orchidaceae (Table 5) has yet to be assessed.

This survey also demonstrated that the assumption that larger hills harbour greater biodiversity (Clements et al. 2008) is not supported for plant species (Table 4). Instead, the diversity of microhabitats is a more important determinant of species-richness (Kiew \& Rafidah 2016). Understanding the ecology of the limestone flora, its microhabitats and niches is crucial to obtain the most complete species checklist. Vermeulen \& Whitten (1999) had contended that it was unlikely that site endemics were restricted to just a small part of a hill, so suggested that protecting even part of a hill can conserve site endemics. However, for plants, many site endemics have small populations, and are specific to microhabitats with restricted areas. For example, Impatiens chikuensis that is only found on screes of loose boulders at the base of the karst hill, a microhabitat that was only found on Ktn 67, or on Batu Caves where Raphidophora burkillii grows on a deeply shaded, steep scree, or Meiogyne kanthanensis on Gunung Kanthan that grows in a deeply shaded gully on relatively deep soil.

Maintaining the integrity of limestone hills is vital to protect sensitive microhabitats and safeguard species richness. The IUCN Guidelines emphasise the importance of having clear legal boundaries (Watson et al. 1997). Davison \& Kiew (1990) stipulated the importance of a buffer zone to protect against fire, which is used in land clearing or weed control. The damage caused by burning is evident in many of the
Chiku hills, where the limestone forest has been cleared right up to the cliff face and many rock faces are still dominated by the rampant climbers that invaded after burning twenty years ago.

\section{Implications for conservation: from the Chiku experience to general guidelines}

The demand for cement and limestone products continues to increase but no state in Malaysia has a holistic plan or strategy to balance commercial needs with other values of karst limestone, whether for biodiversity conservation, geological, landscape, cultural or recreational values. Calls for states to rank hills so that those with highest biodiversity importance and other values are totally protected are beginning to gain a hearing, while the mechanism for issuing mining licenses can be rapid, obtaining protected status is a lengthy and uncertain process. ChemsainKonsultant (2015), suggested that Ktn 49 should be protected with National or State Park status to conserve the biodiversity lost from Ktn 66 and 67. However, it was not specified as who, how or when it should be done, or if it should be a condition of the mining licence to be issued. The status of Ktn 49, 50 and 64 therefore remains uncertain.

The first crucial step in developing a holistic strategy for the exploitation/conservation of limestone at the state level is, as Corlett (2016) emphasised, to close the knowledge gap. This requires detailed surveys that are robust, independent and competent without conflict of interest (Birdlife et al. 2014). The present system in Malaysia, where the EIAs are carried out by consultants appointed and paid for by the project proponent, often results in less than satisfactory assessments. It goes without saying that these surveys should be carried out before the mining license is granted. In addition, the possibility of exploiting subsurface limestone should also be considered.

Surveys need to be carried out by specialists who know how to access the hills, identify and cover all microhabitats, and recognise rare and endangered species. For EIAs of limestone hills, it should be a requirement to re-locate rare species, especially site endemics, and to assess their conservation status. Environmental Impact Assessment (EIA) reports have uniformly 
failed in this respect, the salient example being the recent extinction of Paraboea bakeri (Gesneriaceae) in the wild (Rafidah \& Tan 2012). To avoid this omission in future, the FRIM team has published a list of limestone species known from less than five hills and their localities (Kiew et al. 2017). In addition, if specialists conduct EIAs it is likely that new species will be discovered because the limestone flora is still incompletely known.

Surveys need to be intensive and cover all parts of the hills so that all microhabitats are investigated. In addition, all adjacent hills need to be surveyed, as the Chiku survey has clearly demonstrated that there are great differences in species composition between nearby hills. In the case of the Chiku hills, the assumption that Ktn 49 (Chiku 4) would conserve the biodiversity of Ktn 66 and 67 (Chiku 7 and 8) is shown to be incorrect.

Similarly, suggestions that larger hills conserve higher diversity than small sites or that site endemics are expected to be widespread on a particular hill are shown to be incorrect for plant species.

As is common in EIA reports, Chemsain Konsultant (2015) suggested that plants from Ktn 66 and 67 could be rescued and grown in nurseries with the ultimate aim of being replanted elsewhere. In theory, plant rescue, ex situ conservation and rehabilitation are attractive ideas. In general, limestone plants are difficult to grow because they are adapted to live in specific, narrow microhabitats. Planting protocols need to be developed on a species-by-species basis for ex situ conservation to be successful. It requires horticultural specialists and investment in long-term funding and facilities. As for rehabilitation, quarries abandoned for many years have not been recolonised to any extent and those species that do manage to gain a toehold are secondary forest or weed species (Kiew \& Rafidah 2016). In fact, Birdlife et al. (2014) consider it is impossible to restore limestone vegetation.

\section{CONGLUSIONS}

FRIM survey of four FELDA Chiku hills, Ktn 49, 64, 66 and 67, and Ktn 50 in Relai FR collected 266 species of vascular plant representing $22 \%$ of the limestone flora of Peninsular Malaysia. About $10.2 \%$ of species are endemic to
Peninsular Malaysia, 6 are endemic to Kelantan and three are site endemics, i.e. known only from these limestone hills, of which two are new species discovered during the survey.

For the first time, it is demonstrated that adjacent hills within the same area, each have their own assemblage of species and that no single hill supports more than $50 \%$ of the total Chiku limestone flora. The suggestion that protecting Ktn 49 as a State or National Park would conserve the biodiversity (species richness) of the two hills, Ktn 66 and 67, that will be quarried is flawed because Ktn 49 is home to only $37.2 \%$ of the Chiku species. In fact, 84 species of the 266 species are known only in Ktn 66 and 67 . The survey also showed that hill size measured by basal area is not directly correlated with plant species richness.

Because each of the hills harbours a different assemblage of species, Ktn 49, 50 and 64 should all be totally protected by a minimum of State Park status. Their boundaries should be clearly demarcated and they need to be surrounded by an adequate buffer zone. Their protection needs to be enforced so that further degradation by plantation activities, grazing or poaching be prevented. Both the IUCN (Watson et al. 1997) and the Birdlife et al. (2014) guidelines emphasises the need for a robust Environmental Management System linked to a dedicated Biodiversity Action Plan with a mechanism for its implementation and enforcement.

In Malaysia, conservation planning for limestone karst hills generally lacks a scientific basis. Because individual hills are home to only a fraction of the Peninsula's limestone flora and each hill is home to a different combination of species, it is necessary for each hill to have a detailed, competent survey that focuses on re-finding site endemics, rare and endangered species. At present, biodiversity studies are often rear-guard efforts to salvage what information and specimens is possible before it is consumed by a quarry. However, once scientific data are available, state-wide holistic plans for conservation and exploitation can be developed, incorporating information on karst endemic and endangered taxa into guidelines for selection of those hills that are species rich and have site endemics that require priority total protection, from those hills with little biodiversity value that can be released for commercial exploitation. 


\section{ACKNOWLEDGEMENTS}

The botanical team would like to thank FELDA for permission to carry out the botanical surveys, to the Forest Department of Peninsular Malaysia and the Forest Department of Kelantan for permission to carry out plant collection in their forest reserve. The authors are indebted to field staff Mohd-Hairul MA, Mohd-Nazri A, AmirulSyafiq Y, Noor-Neknazrul H, MuhammadFakrullah HH, Kueh HL and to the nature guides, Che-Asfaizul CR from FELDA, Pak-Lan and Saberi $\mathrm{Z}$ who helped in the field ensuring the success of the botanical surveys. The authors are grateful for the help in specimen identification from Peter OB (Orchidaceae), Saw LG (Palmae), the late Kamarudin S (trees including sterile voucher specimens) and Norzielawati S (Araceae). The botanical surveys were funded by the Malaysian Government through the "Dokumentasi dan Konservasi Biodiversiti Demi Kesejahteraan Hutan dan Kemampanan Sumber Semulajadi (Fasa 1): Subproject 1 Diversiti, Dokumentasi dan Status Konservasi Tumbuhan bagi Pengurusan Sumber Hutan Secara Mampan di Malaysia (SPPII No. P23085100018003)" and "Towards conservation strategy/policy for limestone hills in Peninsular Malaysia: Understanding and documenting plant biodiversity focus on Kelantan and Perlis limestone hill (Phase 1)" under National Conservation Trust Fund (NCTF).

\section{REFERENCES}

BirdLife/FFI/IUCN/WWF. 2014. Joint Briefing Paper on Extraction and Biodiversity in Limestone Areas. BirdLife/FFI/IUCN/WWF, Cambridge.

Bridson D \& Forman L. 1992. The Herbarium Handbook. Revised edition. Royal Botanic Gardens, Kew.

Chemsain-Konsultant SDn. BHD. 2015. Detailed Environmental Impact Assessment for the Proposed Integrated Cement Plant in Mukim Chiku, Gua Musang, Kelantan Darul Naim, Chemsain-Konsultant Sdn. Bhd., Selangor.

CHIN SC. 1977. The limestone hill flora of Malaya 1. The Gardens' Bulletin Singapore 30: 165-219.

Chin SC. 1979. The limestone hill flora of Malaya 2. The Gardens' Bulletin Singapore 32: 64-203.

Chin SC. 1983a. The limestone hill flora of Malaya 3. The Gardens' Bulletin Singapore 35: 137-190.

ChIN SC. 1983b. The limestone hill flora of Malaya 4. The Gardens'Bulletin Singapore 36: 31-91.

Chung RCK, Phoon SN, Chew MY \& Ummul-Nazrah AR. 2009. Buku Panduan Teknik Pengurusan Herbarium Kepong FRIM, Kepong.

Clements R, PkL NG \& et Al. 2008. Using biogeographical patterns of endemic land snails to improve conservation planning for limestone karsts. Biological Conservation 141: 2751-2764.

CoRLETT R. 2016. Plant diversity in a changing world: status, trends and conservation needs. Plant Diversity. 1: 11-18.

Corner EJ. 1988. Wayside Trees of Malaya, Malayan Nature Society, Kuala Lumpur.

Crowther J. 1982. Ecological observations in a tropical karst terrain, West Malaysia. I. Variations in topography, soils and vegetation. Journal of Biogeography 9: 65-78.

Darbyshire I, Anderson S, Asatryan A et al. 2017. Biodiversity and Conservation 26: 1767.

DAvison GWH \& KIEW R. 1990. Survey of Flora and Fauna of Limestone Hills in Kelantan with Recommendations for Conservation. Malayan Nature Society, Kuala Lumpur. Unpublished report.

Henderson MR. 1939. The flora of the limestone hills of the Malay Peninsula. Journal of the Malayan Branch of the Royal Asiatic Society 17, 1: 13-87.

IuCN. 2012. IUCN Red List Categories and Criteria: Version 3.1. Second Edition. IUCN, Gland, Switzerland and Cambridge.

Kiew R, Chung RCK, Saw LG, Soepadmo E \& Boyce PC. 2010. Flora of Peninsular Malaysia Series II: Seed Plants, Volume 1. Forest Research Institute Malaysia, Kepong.

Kiew R, Ghung RCK, Saw LG, Soepadmo E \& Boyce PC. 2011. Flora of Peninsular Malaysia Series II: Seed Plants. Volume 2. Forest Research Institute Malaysia, Kepong.

Kiew R, Chung RCK, Saw LG \& Soepadmo E. 2012. Flora of Peninsular Malaysia Series II: Seed Plants. Volume 3. Forest Research Institute Malaysia, Kepong.

Kiew R, Chung RCK, Saw LG \& Soepadmo E. 2013. Flora of Peninsular Malaysia Series II: Seed Plants. Volume 4. Forest Research Institute Malaysia, Kepong.

Kiew R, Chung RCK, Saw LG \& Soepadmo E. 2015. Flora of Peninsular Malaysia Series II: Seed Plants. Volume 5. Forest Research Institute Malaysia, Kepong.

Kiew R, Chung RCK, Saw LG \& Soepadmo E. 2017. Flora of Peninsular Malaysia Series II: Seed Plants. Volume 6. Forest Research Institute Malaysia, Kepong.

KIEW R. 1991. Limestone Flora. In: R. KIEW (ed.) The State of Nature Conservation in Malaysia. Malayan Nature Society, Kuala Lumpur.

KIEw R. 1997. The Malaysian Highlands and Limestone Hills: Threatenend Ecosystems. State of the Environment in Malaysia. Consumers Association of Penang, Penang.

KIEw R. 2005. Begonias of Peninsular Malaysia. Natural History Publications (Borneo) in Association with Singapore Botanic Gardens, National Parks Board, Singapore.

KIEw R. 2014. Checklist of vascular plants from Batu Caves, Selangor, Malaysia. Check List 19: 1420-1429.

KIEW R. 2016. Impatiens (Balsaminaceae) species from karst limestone in Kelantan, Malaysia, including three new species. Gardens' Bulletin Singapore 68: 225-238.

Kiew R \& Rafidah AR. 2016. Consultancy Report on Rapid Biodiversity Assessment Batu Caves, Selangor for Selangor State Government, Selangor (unpublished). 
Kiew R, Rafidah AR, Ong PT \& Ummul Nazrah AR. 2017. Rare limestone plants in Peninsular Malaysia - what are they, where do they grow and how to conserve them? Malaysian Naturalist 71: 33-41.

Kiew R, Tan JPC, Kamarudin S, Yong KT \& Imin K. 2014. An uncertain future for the plants of Gunung Kanthan, Perak, Malaysia. Cave and Karst Science 41: 120-128.

LARSEN K \& LARSEN SS. 1982. Notes on some Asian Bauhinia. Nordic Journal of Botany 2: 329-332.

Liew TS, Price L \& Clements GR. 2016. Using Google Earth to improve the management of threatened limestone karst ecosystems in Peninsular Malaysia. Tropical Conservation Science 9: 903-920.

MNS (Malaysian Nature Society). 1991. A Conservation Assessment of Limestone Hills in Kinta Valley. Malaysian Nature Society, Kuala Lumpur.

MYBIS (Malaysian Biodiversity Information System). 2019. www.mybis.gov.my

National Physical Plan 2. 2010. Federal Department of Town and Country Planning. Ministry of Housing and Local Government, Kuala Lumpur.

National Policy on Biological Diversity 2016-2025. 2016. Ministry of Natural Resources and Environment (NRE), Putrajaya.

NG FSP. 1978. Tree Flora of Malaya. Volume 3. Longman Group Limited, Kuala Lumpur.

NG FSP. 1989. Tree Flora of Malaya. Volume 4. Longman Group Limited, Kuala Lumpur.

Ong PT, O'Byrne P, SAw LG \& Chung RCK. 2017. Checklist of Orchids of Peninsular Malaysia. Research Pamphlet No. 136. Forest Research Institute Malaysia.

Parris BS, Kiew R, Chung RCK \& SaW LG. 2010. Flora of Peninsular Malaysia Series I: Ferns and Lycophytes. Volume 1. Forest Research Institute Malaysia, Kepong.

Parris BS, Kiew R, Ghung RGK \& SAW LG. 2013. Flora of Peninsular Malaysia Series I: Ferns and Lycophytes. Volume 2. Forest Research Institute Malaysia, Kepong.

Price L. 2014. Cave and Karst of Peninsular Malaysia. A Register. $2^{\text {nd }}$ Edition. Berliner Höhlenkundliche Berichte Volume 54, Berlin.

RAFIDAH AR \& HARON NW. 2013. Microchirita ruthiae sp. nov. (Gesneriaceae) from Peninsular Malaysia. Nordic Journal of Botany 31: 612-615.

Rafidah AR \& TAN JPC. 2012. Paraboea bakeri. Flora of Peninsular Malaysia Online Newsletter 108. http:/ / www.tfbc.frim.gov.my.

Ridley HN. 1922. The Flora of Malay Peninsula. Volume 1. L.Reeve \& Co, London.

Ridley HN. 1923. The Flora of Malay Peninsula. Volume 2. L.Reeve \& Co, London.

Ridley HN. 1924a. The Flora of Malay Peninsula. Volume 3. L.Reeve \& Co, London.

Ridley HN. 1924b. The Flora of Malay Peninsula. Volume 4. L.Reeve \& Co, London.

Ridley HN. 1925. The Flora of Malay Peninsula. Volume 5. L.Reeve \& Co, London.
Rusea G, Latiff A \& Jaman R. 2004. Two new species of Tectaria Cav. (Dennstaedtiaceae) in Peninsular Malaysia. Folia Malaysiana 5: 21-34.

SAW LG. 2010. Vegetation of Peninsular Malaysia. Pp 21-45 in Kiew R et al. (eds) Flora of Peninsular Malaysia, Series II: Seed Plants 1. Forest Research Institute Malaysia, Malaysia.

Saw LG, Chua LSL \& Abdul Rahim N. 2009. Malaysia National Strategy for Plant Conservation. Ministry of Natural Resources and Environment and Forest Research Institute Malaysia, Selangor.

Seidenfaden G \& Wood JJ. 1992. The Orchids of Peninsular Malaysia and Singapore. Olsen \& Olsen, Fredensborg.

Sinar Harian Online. 2014. Kilang simen terbesar bakal dibuka. http://www.sinarharian.com.my/ edisi/kelantan/kilang-simen-terbesar-bakaldibuka-1.313627

TAN CL. 2015. Quarrying threatens to flatten this wildlife refuge in Kelantan. https://www.star2. com/living/living-environment/2015/07/13/ quarrying-threatens-to-flatten-this-wildliferefuge-in-kelantan/

THIERs B. 2017. Index Herbariorum: A Global Directory of Public Herbaria and Associated Staff. Continuously Updated. New York Botanical Garden's Virtual Herbarium, New York.

TURner IM. 1997. A catalogue of the vascular plants of Malaya, Garden's Bulletin Singapore 47: 1-757.

Ummul-Nazrah AR, Mohd Hairul MA, Imin K, Kiew R \& Ong PT. 2018. Vatica najibiana (Dipterocarpaceae), a new species from limestone in Peninsular Malaysia. PhytoKeys 98: 99-106.

UtusAn OnLINE. 2015. Kelantan jamin kilang simen tidak jejas khazanah alam. http://www.utusan.com.my/ berita/wilayah/kelantan-jamin-kilang-simen-tidakjejas-khazanah-8232-alam-1.122129.

Vermeulen JJ \& Whitten T. 1999. Biodiversity and Cultural Property in the Management of Limestone Resources - Lessons from East Asia. World Bank Group, Washington.

Watson J, Hamilton-smith E, Gilieson d \& Kiernan K. 1997. Guidelines for Cave and Karst Protection. IUCN, Gland, Switzerland \& Cambridge.

Weber A. 1997. New taxa of Monophyllaea (Gesneriaceae) from Peninsular Malaysia. Beitrage zur Biologie der Pflanzen 70: 405-406.

Whitmore TC. 1972. Tree Flora of Malaya,Volume 1. Longman Malaysia Sdn Bhd, Kuala Lumpur.

Whitmore TC. 1973. Tree Flora of Malaya, Volume 2. Longman Malaysia Sdn Bhd, Kuala Lumpur.

Xu ZR \& BurtT BL. 1991. Towards a revision of Paraboea (Gesneriaceae) 1: Notes Royal Botany Garden Edinburgh 48: 1-18.

Zulhazman H, Norzielawati S \& Boyce PC. 2017. Studies on the Alocasia clade (Araceae) of Peninsular Malaysia 1: Alocasia farisii sp. nov. from limestone in Kelantan. Nordic Journal of Botany 35: 1-5. 